\title{
Carpotarsal osteochondromatosis
}

INSERM

\section{Source}

INSERM. (1999). Orphanet: an online rare disease and orphan drug data base.

Carpotarsalosteochondromatosis. ORPHA:2767

Carpotarsal osteochondromatosis is a very rare primary bone dysplasia disorder characterized by abnormal bone proliferation and osteochondromas in the upper and lower limbs. 